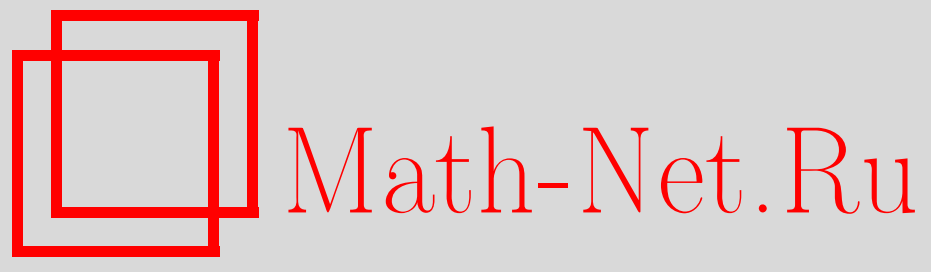

Е. В. Щепин, Код пересечений, УМН, 2013, том 68, выпуск $6,177-178$

DOI: https://doi.org/10.4213/rm9550

Использование Общероссийского математического портала Math-Net.Ru подразумевает, что вы прочитали и согласны с пользовательским соглашением http://www . mathnet.ru/rus/agreement

Параметры загрузки:

IP: 54.196 .121 .252

26 апреля 2023 г., 18:08:54

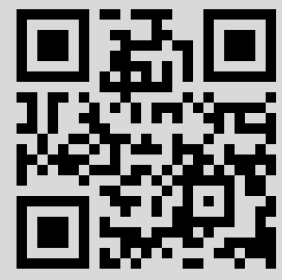




\section{Код пересечений}

\section{Е. В. Щепин}

Мы всегда рассматриваем плоскость с фиксированной декартовой системой координат и считаем, что плоскость расположена так, что ось абсцисс направлена горизонтально, а ось ординат - вертикально.

Изотонным мы будем называть сохраняющий ориентацию гомеоморфизм плоскости, который переводит горизонтальные прямые в горизонтальные прямые и сохраняет вертикальный порядок точек плоскости, т. е. отношение "выше-ниже".

В [1] инварианты изотонных гомеоморфизмов ( $Y$-эквивалентностей) выражались в терминах критических точек функции высоты. В настоящей статье аналогичные результаты получаются на основе так называемого ПРС-кода, примененного еще в программе оптического распознавания CRIPT [1] и объявленного в препринте автора [2].

Область мы всегда предполагаем компактным топологическим многообразием с краем, т. е. имеющей границу, которая является объединением конечного числа непересекающихся простых замкнутых кривых.

Пусть пересечение горизонтальной прямой $L$ и границы замкнутой области $D$ coстоит из конечного числа точек $C_{1}, \ldots, C_{n}$, упорядоченных слева направо (в порядке возрастания абсциссы). Мы будем говорить, что граница области $D$ трансверсально пересекает прямую $L$ в точке $C_{i}$, если всякая окрестность $C_{i}$ содержит точки границы $D$, расположенные по разные стороны от $L$. В противном случае все точки границы из достаточно малой окрестности точки пересечения расположены либо выше, либо ниже прямой $L$. В первом случае мы будем говорить, что в $C_{i}$ область $D$ касается прямой сверху, а во втором - что она касается ее снизу. Договоримся кодировать тип пересечения следующим образом: точку трансверсального пересечения русской буквой "П" (продолжение), компоненту касания снизу - русской буквой "Р" (рождение), компоненту касания сверху - русской буквой "С" (смерть). Таким образом, пересечение прямой и области кодируется словом трехбуквенного алфавита, которое мы называем ПРС-кодом пересечения.

Горизонтальная прямая $L$ называется критической для области $D$, если код ее пересечения с областью содержит рождение или смерть. ПРС-код области с конечным числом критических значений определяется как последовательность отделенных пробелами ПРС-кодов ее критических уровней, в порядке сверху вниз.

Определим верхнюю $\mathrm{val}^{+}$и нижнюю $\mathrm{val}^{-}$валентности кода следующим образом: $\operatorname{val}^{+}(\mathrm{P})=\operatorname{val}^{-}(\mathrm{C})=0, \operatorname{val}^{-}(\mathrm{P})=\operatorname{val}^{+}(\mathrm{C})=2, \operatorname{val}^{+}(\Pi)=\operatorname{val}^{-}(\Pi)=1$.

Лемма 1. Пусть $A_{1} \ldots A_{n}$ и $B_{1} \ldots B_{m}$ представляют собой ПРС-кодъ пересечения некоторой области $D$ с горизонталями уровней $y_{1}$ и $y_{2}$ соответственно, так что в интервале $\left(y_{1}, y_{2}\right)$ область $D$ критических значений не имеет. Тогда $\sum_{i=1}^{n} \operatorname{val}^{+}\left(A_{i}\right)=\sum_{i=1}^{m} \operatorname{val}^{-}\left(B_{i}\right)$.

ДокАзАТЕЛЬСтво. Пересечение границы области $D$ с горизонтальной полосой диапазона $\left[y_{1}, y_{2}\right]$ является объединением некоторого количества дуг $C_{1}, \ldots, C_{k}$, концы которых лежат на разных (верхней и нижней) границах полосы. Точка границы полосы, имеющая код "П", является концом ровно одной из дуг $C_{i}$. В точках типа "Р" верхней границы полосы и в точках типа "С" нижней границы полосы сходятся по две таких дуги. Таким образом, мы видим, что количество дуг $C_{i}$, которым принадлежит данная точка верхней (нижней) границы полосы, совпадает с нижней (верхней)

Работа выполнена при поддержке РФФИ (грант № 11-01-00822) и Правительства РФ (постановление № 220, дог. № 11.G34.31.0053).

DOI: $10.4213 / \mathrm{rm} 9550$ 
валентностью ее кода. Поэтому сумма верхних валентностей кодов точек нижней границы полосы, так же как и сумма нижних валентностей кодов точек верхней границы полосы, совпадает с $k$ - количеством этих дуг. Лемма доказана.

Занумеруем дуги $C_{i}$ в том порядке (слева направо), в котором расположены точки их пересечения со средней линии рассматриваемой полосы. Так как различные дуги внутри полосы не пересекаются, то порядок, в котором располагались их пересечения с горизонталью, одинаков для всех внутренних горизонталей полосы. На границе полосы концы соседних дуг могут совпадать, что и происходит в точках смертей нижней границы полосы и в точках рождений верхней. Коды $A_{v}$ и $B_{u}$ конца дуги $C_{i}$ на верхней границе полосы определяются на основе подсчета соответствующих валентностей по правилам

$$
u=\min \left\{k \mid \sum_{l=1}^{k} \operatorname{val}^{-}\left(B_{l}\right) \geqslant i\right\}, \quad v=\min \left\{k \mid \sum_{l=1}^{k} \operatorname{val}^{+}\left(A_{l}\right) \geqslant i\right\} .
$$

Теорема 1. Пусть области $D_{1}$ и $D_{2}$ имеют конечное число критических уровней и конечные пересечения с горизонталями. Тогда их ПРС-коды совпадают в том и только том случае, когда существует изотонный гомеоморфизм плоскости, переводящий одну из них в другую.

ДокАЗАТЕЛЬСтво. Совпадение ПРС-кодов изотонных областей очевидно. В обратном направлении, нетрудно построить изотонный гомеоморфизм, который переводит все критические горизонтали одной области в критические горизонтали другой, ибо количества этих горизонталей для областей с совпадающими ПРС-кодами совпадают. Поэтому в дальнейшем мы будем считать, что критические уровни рассматриваемых областей совпадают.

На каждом критическом уровне $y_{i}$ совпадение ПРС-кода позволяет установить взаимно однозначное, сохраняющее тип пересечения и горизонтальный порядок соответствие между точками пересечений границ рассматриваемых областей. Это соответствие нетрудно "по линейности" на каждом уровне продолжить до сохраняющего высоту изотонного гомеоморфизма плоскости. Поэтому, не теряя общности, мы можем предполагать, что пересечения рассматриваемых областей с критическими горизонталями совпадают. Для некритической горизонтали количество точек пересечения с границей области определяется, как было показано выше, на основе подсчета валентностей кодов ближайшего критического уровня. Поэтому для рассматриваемых областей эти числа совпадают и равны числу компонент пересечения границ областей с соответствующей полосой. Также было показано, что если перенумеровать компоненты в порядке возрастания абсциссы их пересечения с какой-либо промежуточной горизонталью, то компоненты с одинаковыми номерами будут иметь совпадающие концы. Поэтому следующий гомеоморфизм горизонтали $L$ непрерывно зависит от ее уровня: $i$-й слева точке пересечения границы $D_{1}$ с $L$ ставится в соответствие $i$-я слева точка пересечения границы $D_{2}$ с $L$, на остальные точки прямой $L$ гомеоморфизм продолжается линейно. Совокупность определенных таким образом гомеоморфизмов горизонталей, пересекающих рассматриваемые области, и тождественных гомеоморфизмов горизонталей, не пересекающих их, и определяет искомый изотонный гомеоморфизм плоскости. Теорема доказана.

\section{Список литературы}

[1] G. M. Nepomnyashchii, E. V. Shchepin, Int. J. Imag. Syst. Tech., 3:3 (1991), 213-221. [2] E. Shchepin, A topological code for plane images, arXiv: 1212.0819 .

\section{Евгений Витальевич Щепин}

(Evgeny V. Shchepin)

Математический институт им. В. А. Стеклова РАН;

Ярославский государственный университет

E-mail: scepin@mi.ras.ru
Представлено В. М. Бухштабером Принято редколлегией 05.09 .2013 